Check for updates

Cite this: Mater. Adv., 2021, 2,6083

Received 5th July 2021, Accepted 23rd August 2021

DOI: 10.1039/d1ma00579k

rsc.li/materials-advances

\section{Synthesis of carbon nitride oligomer as a precursor of melon with improved fluorescence quantum yield $\dagger$}

\author{
Yuto Miyake, ${ }^{a}$ Goichiro Seo, ${ }^{a}$ Kotaro Matsuhashi, ${ }^{a}$ Noriyuki Takadab and \\ Kaname Kanai iD *a
}

\begin{abstract}
Carbon nitride ( $\mathrm{CN}$ ) compounds have attracted significant attention as new photocatalysts capable of decomposing water and producing hydrogen upon irradiation using visible light. To date, many studies have focused on their applications as photocatalysts. Melon, a typical CN polymer, is a semiconductor with a direct energy gap, because of which it cannot exhibit high photocatalytic activity without a cocatalyst. However, melon shows high potential as a blue-light-emitting material with high color purity, and the basic physical characteristics of melon are worth clarifying. Additionally, the synthesis of melon during thermal polymerization remains unclear. In this study, the polymerization of melon is investigated by varying the thermal polymerization conditions, and new $\mathrm{CN}$ compounds with optical properties similar to those of melon are examined. Characterization of the products prepared under different conditions allowed the identification of a new CN compound, a likely precursor of melon, at temperatures slightly below the calcination temperature of melon. This new compound, which consists of oligomers with four melem units, is very stable in air and shows improved optical properties compared to those of melon.
\end{abstract}

\section{Introduction}

The use of fossil fuels as energy sources has resulted in natural resource depletion and environmental degradation. Renewable energy is a promising alternative to reduce the use of fossil fuels. Hydrogen is a renewable and sustainable clean energy source to generate electricity using fuel cells, and many researchers have investigated efficient hydrogen generation methods. Extensive research on photocatalytic hydrogen evolution was initiated after the publication of a report in 1972, describing the electrochemical photolysis of water over $\mathrm{TiO}_{2}{ }^{1}{ }^{1}$ However, the energy band gap exceeds $3 \mathrm{eV}$ for all polymorphs of $\mathrm{TiO}_{2}$; thus, ultraviolet irradiation at wavelengths shorter than $400 \mathrm{~nm}$ is necessary to afford photoactivity. Several new photocatalyst materials have been identified for replacing $\mathrm{TiO}_{2} \cdot{ }^{2-5}$ Among these materials, graphitic carbon nitride (g-CN) has attracted significant attention as a promising candidate because it is metal-free and composed of only carbon and nitrogen, making it an environmentally friendly and

\footnotetext{
${ }^{a}$ Department of Physics, Faculty of Science and Technology, Tokyo University of Science, 2641 Yamazaki, Noda, Chiba 278-8510, Japan.

E-mail: kaname@rs.tus.ac.jp

${ }^{b}$ National Institute of Advanced Industrial Science and Technology, Research Institute for Advanced Electronics and Photonics, Central 2, 1-1-1 Umezono, Tsukuba, Ibaraki 305-8568, Japan

$\dagger$ Electronic supplementary information (ESI) available. See DOI: 10.1039/d1ma00579k
}

sustainable material. The availability of a convenient method for the thermal polymerization of the precursor makes graphitic carbon nitride particularly appealing.

g-CN has a layered structure comprising tri-s-triazine units connected through planar amino groups and exhibits excellent chemical and thermal stability, flexibility, and photoabsorption in the visible range. ${ }^{6,7}$ These properties make g-CN suitable for use in visible-light photocatalysis to generate hydrogen. Additionally, the ability of $\mathrm{g}-\mathrm{CN}$ to adsorb carbon dioxide, heavy metals, and organic materials makes g-CN highly attractive in terms of functionality for various applications. ${ }^{8-10}$

As previously described by several researchers, ${ }^{11,12}$ most $\mathrm{g}^{-} \mathrm{C}_{3} \mathrm{~N}_{4}$ samples, which is a g-CN compound with a stoichiometric ratio of $\mathrm{C}$ to $\mathrm{N}$ is $3: 4$, synthesized to date contain significant amount of hydrogen atoms, as demonstrated by amino group vibrations in the infrared spectra and other experimental evidence. These reports indicate that most $\mathrm{g}-\mathrm{C}_{3} \mathrm{~N}_{4}$ samples prepared via thermal polymerization of the precursor, 2,4,6-triamino-1,3,5-triazine (melamine: Fig. 1), are different compounds. Recent studies have shown that the thermal polymerization of melamine under an inert atmosphere, typically used for the synthesis of $\mathrm{g}$-CN, provides melon and not $\mathrm{g}-\mathrm{C}_{3} \mathrm{~N}_{4}$, based on X-ray diffraction (XRD) and quantitative analysis by X-ray photoemission (XPS). ${ }^{13}$ Melon was identified by Liebig ${ }^{14}$ in 1834, and first synthesized by Berzelius et al. It is a linear polymer composed of a one-dimensional 
<smiles>Nc1nc(N)nc(N)n1</smiles>
melamine

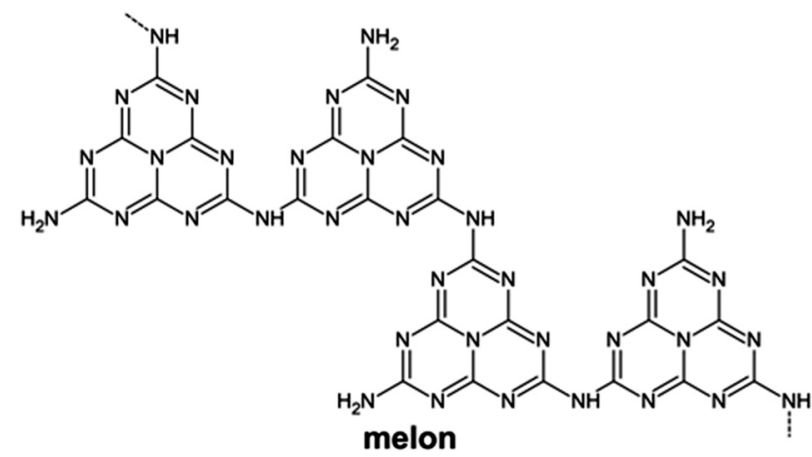

Fig. 1 Molecular structures of melamine, melem, and melon.

chain of 2,5,8-triamino-tri-s-triazine (melem) units, as shown in Fig. 1. The commonly used method for synthesizing melon is thermal polymerization using melamine and dicyandiamide as the starting materials. A comparison of the electronic structures of $\mathrm{g}-\mathrm{C}_{3} \mathrm{~N}_{4}$ and melon shows that the dimensionality of the system has a significant effect on their electronic structures even though these have the same building unit of melem. The energy band calculations of previous studies suggest that melon has a direct gap, which predicts different optical properties from those of $\mathrm{g}-\mathrm{C}_{3} \mathrm{~N}_{4}$ with an indirect gap. ${ }^{15}$ The energy gap, $E_{\mathrm{g}}$, of $\mathrm{g}-\mathrm{C}_{3} \mathrm{~N}_{4}$ is smaller than that of melon because of the higher degree of polymerization of the two-dimensional system. Although melon has a high exciton recombination probability after visible light absorption (in principle), which may limit its photocatalytic activity, many studies have reported the enhancement of its photocatalytic activity. If most of the previously reported materials are melon rather than $\mathrm{g}-\mathrm{C}_{3} \mathrm{~N}_{4}$, as mentioned above, the capability of melon as a photocatalyst is already well-established. Additionally, melon has been identified as a potential material for application in electroluminescent devices owing to its direct gap semiconducting properties. ${ }^{16}$ However, the investigation of the potential of melon as a new organic semiconducting material has been retarded in comparison to its photocatalytic activity. Furthermore, the basic information regarding melon remains insufficient. For instance, although melon can be synthesized relatively easily by thermal polymerization, the dependence of its structure and chemical state on thermal polymerization conditions is yet to be thoroughly examined.

It is well-known that the thermal polymerization of melon starting from melamine involves a monomer intermediate, melem. The present study mainly focuses on the changes in the structures of the products and their optical properties during polymerization from melem to melon. This can allow the identification of polymerization conditions that can lead to an improvement in the photocatalytic activity and other physical properties not only of melon but also CN-based compounds.

In this study, the thermal polymerization conditions are carefully examined, and a new stable solid phase of the melem-oligomerbased compound is identified during the polymerization of melem to melon. The optical properties of the compound are investigated, and it is determined that it possesses fluorescence properties with an absolute quantum yield of approximately twice that of melon. This allows the identification of appropriate polymerization conditions for the application of the CN-based compound as a new lightemitting material.

\section{Experimental}

\subsection{Calcination procedure}

To synthesize melon, melamine (3.0 g, purity: 99.0\% Wako Pure Chem., Ind., Ltd, 139-00945) was placed at the bottom of a quartz tube (Nichiden-Rika Glass Co., Ltd, P-18SM), and the tube was capped with aluminum foil with a pinhole of $\sim 0.6 \mathrm{~mm}$ diameter. The aluminum foil was fixed with a tungsten wire, and thermal polymerization was performed in this semi-closed system. ${ }^{17}$ Melamine was heated at the target temperature $\left(350,415\right.$, or $\left.450{ }^{\circ} \mathrm{C}\right)$ in the quartz tube filled with $\mathrm{N}_{2}$ at atmospheric pressure for a specific time. Prior to heating, the inside of the quartz tube was replaced with $\mathrm{N}_{2}$. After decreasing the temperature to room temperature $\left(\sim 20{ }^{\circ} \mathrm{C}\right)$ at a rate of $2{ }^{\circ} \mathrm{C} \mathrm{min}^{-1}$, the powder product in the quartz test tube was removed and ground in a mortar to afford a uniform particle size.

The procedures under vacuum were performed as follows. An alumina crucible was used for heating under vacuum, and the sample was heated by the resistive heat generated by the tungsten wire wrapped around the periphery of the crucible. The average vacuum during calcination was approximately $1 \times$ $10^{-4} \mathrm{~Pa}$. During heating under vacuum, a thermocouple was inserted into the powder part of the crucible in the chamber to determine the temperature, and the temperature was increased to $180{ }^{\circ} \mathrm{C}$ at a rate of approximately $1.5{ }^{\circ} \mathrm{C} \mathrm{min}{ }^{-1}$. After a predetermined time ( 4.0 or $5.3 \mathrm{~h}$ ), the heating was stopped, and the sample was removed from the chamber after the temperature of the sample decreased to room temperature. The obtained sample was then ground using a mortar and pestle.

\subsection{Analysis methods}

UV-visible (UC-vis) spectroscopy data (V-650 spectrometer, JASCO) for the samples were collected by placing the samples between two quartz plates to combine the transmission and diffuse reflection methods. To measure the absorbance of melem, it was dissolved in DMSO and the absorbance in solution was measured to suppress the effect of sample luminescence. The $\mathrm{KBr}$ pellet method was used to record the Fourier-transform infrared (FT-IR) spectra (FT/IR 6100 spectrometer, JASCO) of the powder samples. XRD analysis was conducted using a diffractometer (Ultima IV, Rigaku) with a $\mathrm{Cu} \mathrm{K} \alpha$ radiation source $(\lambda=0.15496 \mathrm{~nm})$. 
XPS data for the powder samples were collected using an AXISNOVA instrument (KRATOS ANALYTICAL Ltd, UK) with monochromatic $\mathrm{Al} \mathrm{K \alpha}(h \nu=1486.6 \mathrm{eV})$ as the excitation source. The XPS data were analyzed using Voigt functions by employing the XPSPEAK41 software program (written by Raymund W. M. Kwok).

Photoluminescence (PL) spectra for the powder samples were recorded by placing these between two glasses, using a spectrometer (Nanolog, HORIBA) with a Xe light source $(\lambda=$ $350 \mathrm{~nm}$ ). The incident angle of the excitation light was $30^{\circ}$ from the normal direction of the sample surface.

The quantum yield was measured using an absolute PL quantum yield instrument (C9920-02, Hamamatsu Photonics K.K.). The measurements were performed at the excitation light wavelengths of $310 \mathrm{~nm}$ and $340 \mathrm{~nm}$ using a $150 \mathrm{~W}$ Xe lamp as the light source.

Molecular orbital (MO) and energy band calculations were performed using the density functional theory (DFT). The band calculations were performed using the CASTEP plane-wave basis set and the GGA exchange-correlation function. The structures of the compounds were created using the Materials Studio software. Previously reported melem ${ }^{18}$ and melon ${ }^{19}$ structures were used for the calculations.

XRD profiles were calculated for the above-mentioned structures using the Powder Diffraction Pattern package by employing the Visualization for Electronic and Structural Analysis (VESTA) program.

\section{Properties of $\mathrm{CN}$ compounds synthesized at different temperatures}

\subsection{Characterization of $\mathrm{CN}$ compounds synthesized at atmospheric pressure}

When melon was synthesized by thermal polymerization using melamine as a precursor and the calcination temperature was gradually increased, compounds with several different structures were obtained at different temperatures. To determine the structures and chemical states of the products obtained at different calcination temperatures, the XRD and FT-IR data were collected (Fig. 2). These results show that the crystal structures and compositions of products change significantly with a variation in temperature. Detailed calcination temperature dependence of the XRD and FT-IR data is shown in Fig. S1 (ESI $\dagger$ ). Melem was prepared by heating melamine at $400{ }^{\circ} \mathrm{C}$. Melon could be obtained by heating melamine in $\mathrm{N}_{2}$ atmosphere at a relatively high temperature of $430-600{ }^{\circ} \mathrm{C}$. The XRD and FT-IR data of melem and melon are in good agreement with the previously reported data, ${ }^{13,18-22}$ indicating that the compounds are successfully synthesized. The theoretical pressure phase diagrams of these $\mathrm{CN}$ compounds obtained by freeenergy calculations ${ }^{23}$ are consistent with the conditions described here for the synthesis of melem and melon. However, when melamine was heated at $415{ }^{\circ} \mathrm{C}$, the XRD patterns obtained for melem and melon were different. Hereafter, this product is called "as-synthesized CN415 (as-CN415)." As-CN415 shows XRD patterns similar to those of melem, but the characteristic peak observed at $2 \theta \sim 25^{\circ}$, as depicted by the broken line in Fig. 2(a), is not present in either melem or melon. The $d$ spacing calculated from this peak is $0.354 \mathrm{~nm}$. Although there are some reports of compounds affording XRD data similar to those of as-CN415, no reports on structural identification or optical properties have been published. ${ }^{24-26}$ Furthermore, rinsing of as-CN415 with dimethyl sulfoxide (DMSO) to remove residual unreacted melem allowed the separation of products. The main component of the material removed by DMSO is considered to be unreacted melem from the UV-Vis measurements. This is consistent with the FT-IR results. On the other hand, soluble molecular species, which may include partially condensed species along with unreacted melem, should also
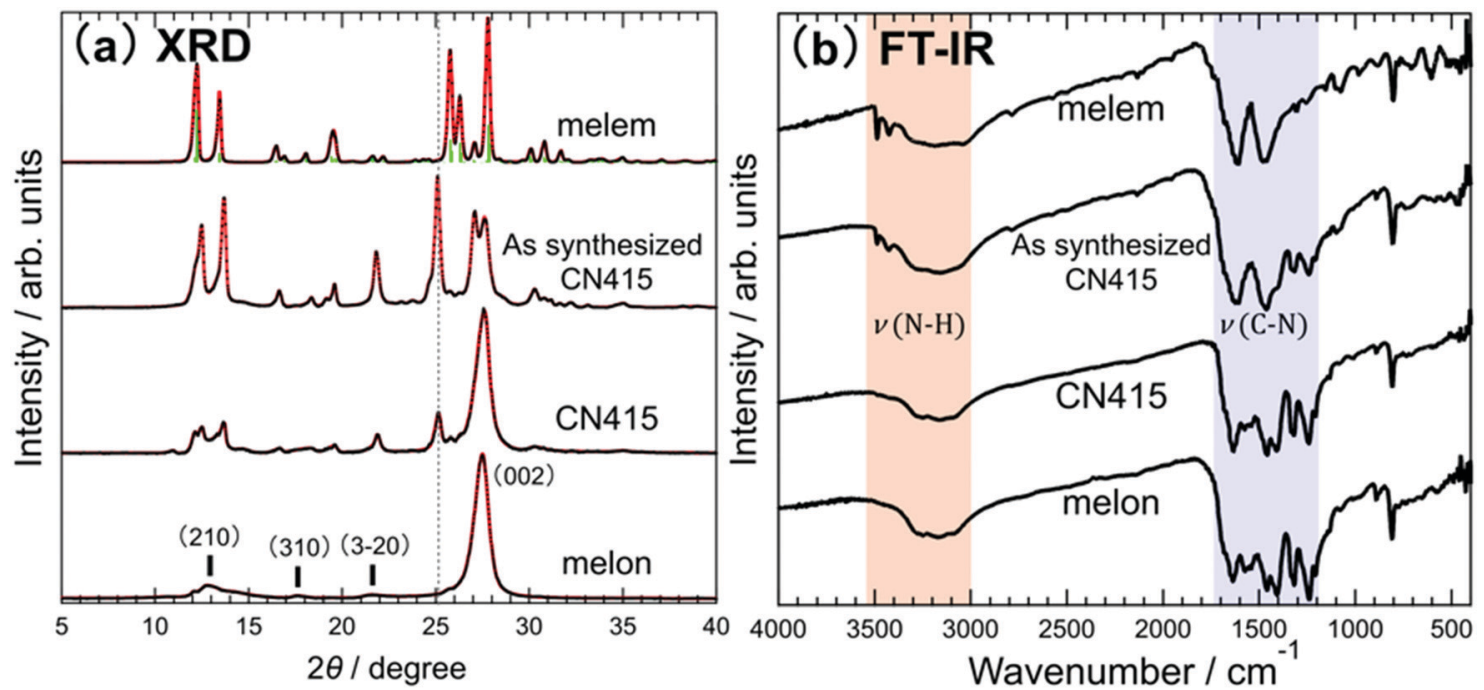

Fig. 2 XRD and FT-IR data of products prepared via calcination under ambient conditions. (a) XRD profiles of powder samples of melem, as-synthesized CN415, CN415, and melon. Melem and melon were prepared at 310 and $450^{\circ} \mathrm{C}$, respectively. As-synthesized CN 415 was prepared at $415^{\circ} \mathrm{C}$. Vertical bars in the data for melem represent simulated diffraction peaks. ${ }^{18}$ (b) Corresponding FT-IR spectra of the samples subjected to XRD analysis. 

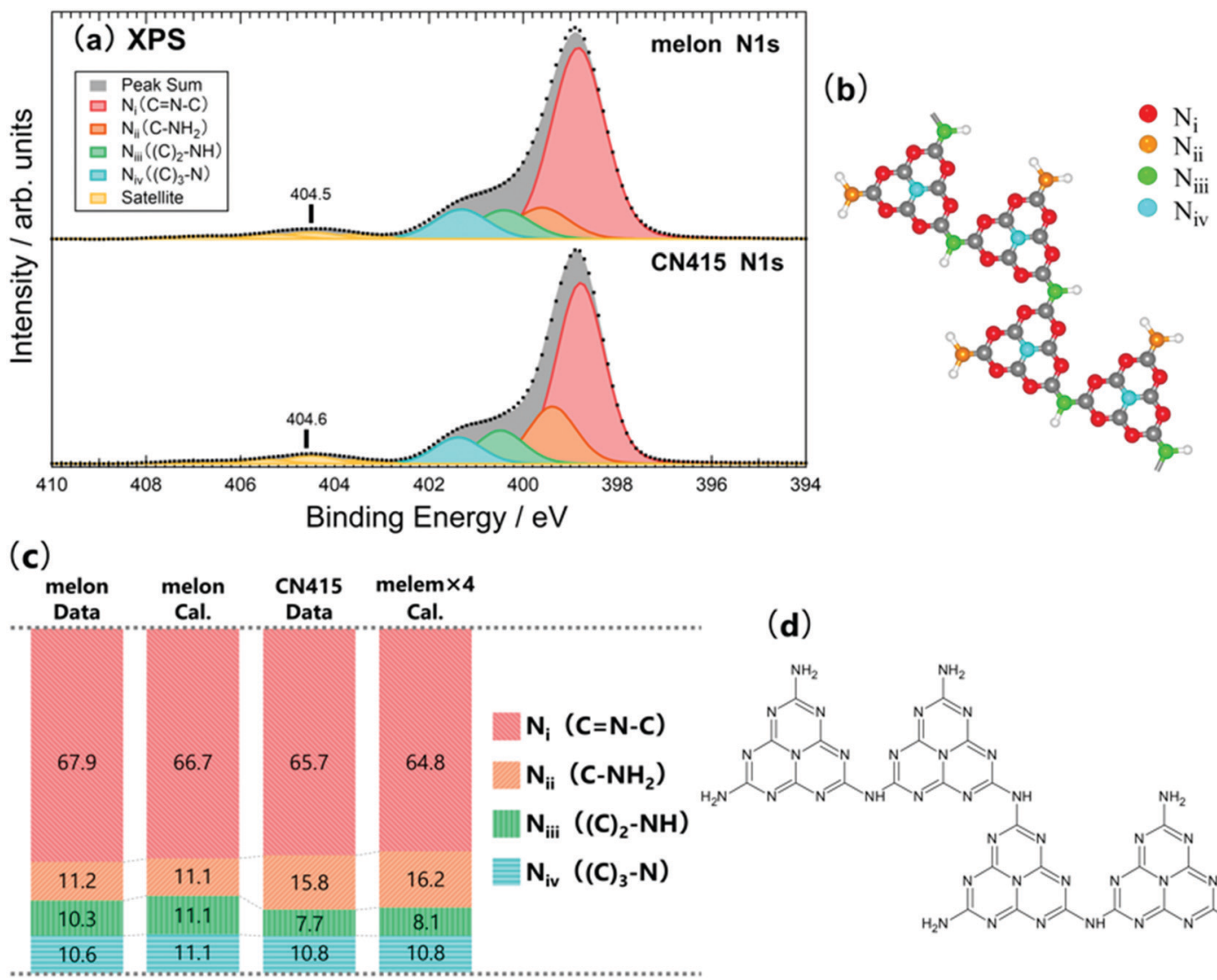

(d)

have been removed. The XRD pattern of this product is similar to that of melon. A peak at approximately $27^{\circ}$, corresponding to the stacked layer structure in the $c$-axis direction that is also observed for melon, is observed, suggesting that it has a layered structure similar to melon. However, in addition to the peak observed at $2 \theta \sim 25^{\circ}$, some differences from the data for melon are also observed. This product is referred to as "CN415," until its identity is clearly established in this paper.

Based on the FT-IR data, the NH stretching vibration peaks $(\nu(\mathrm{N}-\mathrm{H}))$ observed at approximately $3500 \mathrm{~cm}^{-1}$ in the spectrum of melem ${ }^{20}$ indicate the presence of $\mathrm{NH}_{2}$ groups in melem, and identical peaks are also observed in the data for as-CN415. Considering the XRD data, the observation of $\nu(\mathrm{N}-\mathrm{H})$ peaks for as-CN415, similar to melem, indicates that as-CN415 contains residual melem. A comparison of the FT-IR data of as-CN415 to that of melamine ${ }^{27}$ shows that a small amount of unreacted melamine is present in as-CN415. In contrast, the $\nu(\mathrm{N}-\mathrm{H})$ peaks in the spectrum of melon are not observed at the wavenumber at which these are observed in the spectrum of melem. This is due to a partial loss of $\mathrm{NH}_{2}$ groups in melem by thermal polymerization. No clear difference in the FT-IR spectra of melon and CN415 are observed, indicating that their chemical states are similar to each other. Therefore, considering these results in combination with the XRD data, it can be concluded that CN415 may be a polymorph or precursor of melon. The presence of CN415 is not explicitly indicated in the theoretical phase diagram. ${ }^{23}$

XPS data were collected to identify the differences between the chemical states of melon and CN415. The N 1s core level XPS data of the compounds are shown in Fig. 3(a). Based on the FT-IR data shown in Fig. 2(b), it is clear that CN415 has similar functional groups as those of melon. Therefore, the compositions of melon and CN415 were determined by analyzing the peak intensity in the XPS data. First, based on the intensity ratios of the C 1s spectrum shown in Fig. S2 (ESI $\dagger$ ) and the N 1s spectrum in Fig. 3(a), the ratio of the number of carbon atoms to those of nitrogen atoms $(\mathrm{C} / \mathrm{N}$ ratio) in the specimens is found to be almost identical, i.e., 0.66 for melon and 0.65 for CN415. Next, the intensities of the $\mathrm{N}$ components were compared. As shown in Fig. 3(b), there are four types of nitrogen atoms 
contained in the melon structure with distinction chemical environments: $\mathrm{N}_{\mathrm{i}}$ forms the skeleton of the heterocycle, $\mathrm{N}_{\mathrm{ii}}$ forms the terminal amino group, $\mathrm{N}_{\mathrm{iii}}$ forms the bridge connecting the melem units, and $\mathrm{N}_{\mathrm{iv}}$ occupies the center of the heterocycle. The XPS data of melon and CN415 were analyzed by the standard peak-fitting method using four individual peaks with binding energy values of the 1 s orbitals of each nitrogen obtained from previous studies, ${ }^{13}$ and these are summarized in Table S1 (ESI $\dagger$ ). The $\mathrm{N}_{\mathrm{i}}: \mathrm{N}_{\mathrm{iv}}$ ratio, corresponding to the nitrogens constituting the heterocycle, remains almost unchanged in both melon and CN415 (approximately $6: 1$ ). This indicates that CN415 also has a structure consisting of heterocyclic units such as in the case of melon. This is also consistent with the nearly identical $\mathrm{C} / \mathrm{N}$ ratios for melon and $\mathrm{CN} 415$. In contrast, a clear difference between melon and CN415 is observed in terms of the intensity ratio of $\mathrm{N}_{\mathrm{ii}}$ and $\mathrm{N}_{\mathrm{iii}}$. As shown in Fig. 3(c), the intensity ratio of each $\mathrm{N}$ 1s peak in the experimental data of melon shows good agreement with the stoichiometric proportion of melon. In contrast, CN415 has a higher proportion of $\mathrm{N}_{\mathrm{ii}}$, the amino group at the end of the molecular chain, and a lower proportion of $\mathrm{N}_{\mathrm{iii}}$ forming the bridge that connects the melem units, compared to the corresponding proportions in melon. This result indicates that CN415 is an oligomeric form containing a shorter molecular chain than melon, which decreases the nitrogen proportion in the bridge and increases that at the end of the molecular chain. Furthermore, to determine the number of melem units connected in $\mathrm{CN} 415$, the calculated intensity ratio of the $\mathrm{N}$ 1s peak that increased in each melem unit was examined, and four melem units reproduced the observed results. Therefore, CN415 is composed of oligomers with molecular structure constituting four connected melem units (melem tetramer), as shown in Fig. 3(d). In other words, CN415 is speculated to have a lower degree of polymerization than melon and its precursor. The proportions of $\mathrm{N}_{\mathrm{i}}, \mathrm{N}_{\mathrm{ii}}, \mathrm{N}_{\mathrm{iii}}$, and $\mathrm{N}_{\mathrm{iv}}$ calculated from the stoichiometric proportion of oligomers with three and five melem units are $64.3 \%, 17.9 \%, 7.1 \%$, and $10.7 \%$ and $65.2 \%, 15.2 \%, 8.7 \%$, and $10.9 \%$, respectively. The possibility that the building unit of CN415 is a trimer can be ruled out, but the pentamer cannot be completely ruled out. However, when comparing the ratio of $\mathrm{N}_{\mathrm{ii}}$ to $\mathrm{N}_{\mathrm{iii}}$, it is more appropriate to consider it as a tetramer. The likelihood of the model structure shown in Fig. 3(d) as a reasonable possibility will be described later. However, it should be noted that in addition to linear structures such as tetramer, branched structures are also possible candidates for the structure of CN415, as they can reproduce the ratio of $\mathrm{N}_{\mathrm{ii}}$ to $\mathrm{N}_{\mathrm{iii}}$.

The XPS data provide evidence for the chemical states of non-metallic materials as well as $E_{\mathrm{g}}$. A small hump is observed toward the high binding energy side of the main peak in the $\mathrm{N}$ 1s spectra of melon and CN415 (Fig. 3(a)), and this feature is called "shake-up satellite." The shake-up satellite is observed due to single electron excitation accompanied by photoemission from the $\mathrm{N}$ 1s core level. A portion of the kinetic energy of the photoelectron from the core level causes excitation of a single electron in the valence band to the conduction band. Thus, the energy of the shake-up satellite determined from the main peak roughly represents the energy gap, $E_{\mathrm{g}}$, in the presence of the $\mathrm{N}$ 1s core hole. It is generally not possible to determine the correct value of $E_{\mathrm{g}}$ from the shake-up satellite owing to the presence of the core hole following photoemission, but the magnitude of $E_{\mathrm{g}}$ can be estimated. The energy difference between the shake-up satellite and main peak is greater by approximately $0.1 \mathrm{eV}$ in CN415 than that in melon, as shown in Fig. 3(a). Therefore, the $E_{\mathrm{g}}$ value of CN415 is greater than that of melon. This result is consistent with the
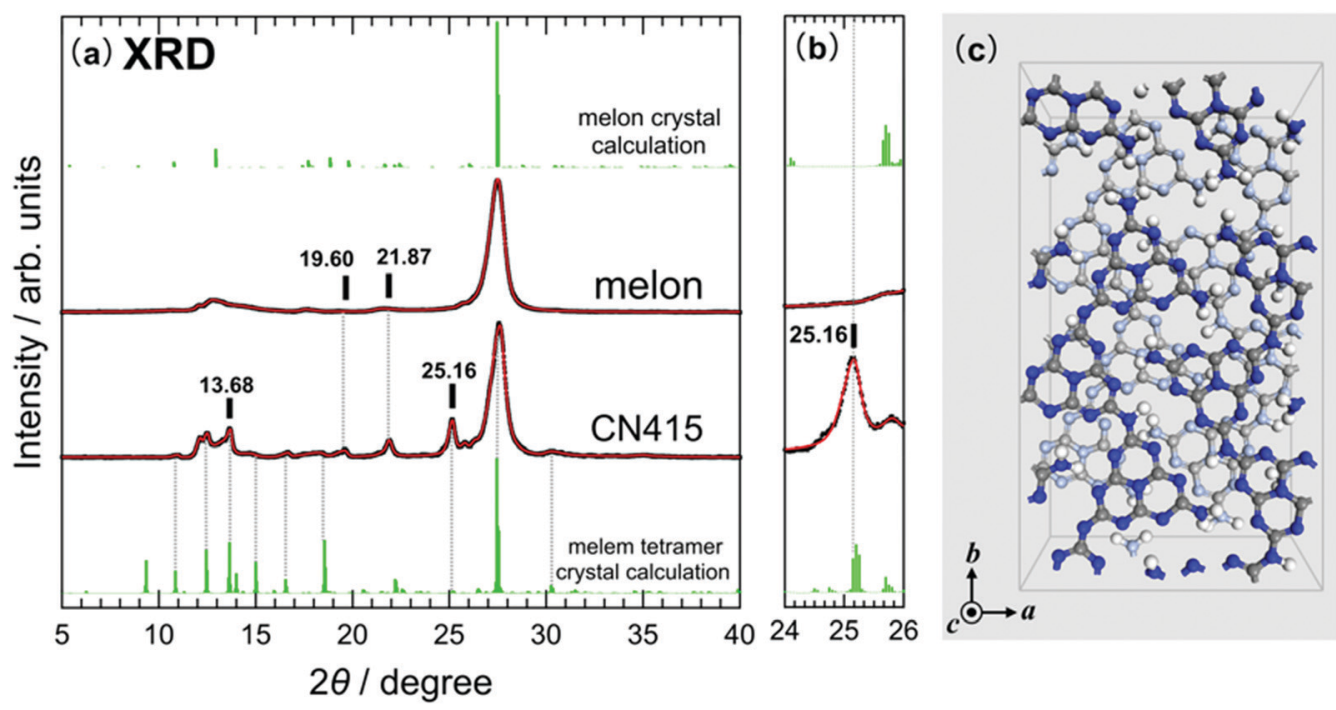

Fig. 4 (a) XRD profiles of melon and CN415 (identical to those in Fig. 2(a)) and calculated XRD patterns for melon and CN415. The results of the XRD simulation for melon and CN415 ("melem tetramer") are also shown. The model structure of CN415 shown in (c) was used for the calculation, which is obtained using the melem tetramer as a building unit. (b) Magnified view of the XRD profiles near $2 \theta=25^{\circ}$. (c) Unit cell of the model structure of $\mathrm{CN} 415$ viewed from the $c$-axis direction. 
conclusion that CN415 consists of oligomers with a shorter molecular chain length compared to melon, based on the peak fitting analysis of the XPS data mentioned above, because the short $\pi$-conjugated length generally affords a large energy gap.

The XPS data suggest that an oligomer consisting of four melem units should be the building unit in the crystal structure of CN415. Using the CN415 crystal structure model in which melem tetramers were arranged, attempts to reproduce the XRD peaks of CN415 shown in Fig. 2(a) were made. Fig. 4(a) shows the results of the XRD simulation for melon and CN415, together with their experimental profiles. The model structure of CN415 shown in Fig. 4(c), which is obtained using the melem tetramer as a building unit, can reproduce the diffraction peaks characteristic of CN415 (atomic coordinates of CN415 are listed in Table S2, ESI $\dagger$ ). For instance, the peaks at $2 \theta$ values of $10.98^{\circ}$, $12.50^{\circ}, 13.68^{\circ}, 14.98^{\circ}, 16.67^{\circ}, 18.50^{\circ}, 25.16^{\circ}$, and $30.27^{\circ}$, which are not observed in the data for melon, can be explained on the basis of the simulation data. Fig. 4(b) shows a magnified view of the XRD profiles near $2 \theta=25^{\circ}$, and the model structure of CN415 shows a peak at $25.16^{\circ}$, which is observed only in CN415. This peak is assigned to the (440) diffraction. As shown in Fig. S3 (ESI $\dagger$ ), the peak observed at around $25^{\circ}$ cannot be reproduced by the model structure prepared using melem pentamer as a building unit. In contrast, the peak at $19.6^{\circ}$ could not be reproduced using the model structure of CN415; this is possibly assigned to be a peak derived from melon, and it is considered that the melon structure partially coexists with CN415. In other words, it is expected that only a small portion of CN415 is changed to the melon structure due to additional polymerization via calcination at $415{ }^{\circ} \mathrm{C}$, implying that CN415 is a melon precursor. This indicates that the crystal structure of CN415 corresponds to the structure that is formed during the transition from the herringbone structure of melem ${ }^{18}$ to its layered structure via thermal polymerization. The reproduction of multiple peak positions in the measured XRD data of CN415 further supports the conclusion that the building unit of the CN415 crystal structure is a melem tetramer. Although this conclusion can be reasonably achieved at this time, additional studies including the reproduction of XRD intensity are required in the future. Hereafter, CN415 is referred to as the "melem tetramer (MT)" in this article.

The band calculation results for melon and MT are shown in Fig. S4 (ESI $\dagger$ ). Some band dispersion can be observed in melon; in particular, the lowest conduction band shows a dispersion along the direction in which the polymer chain extends, and the highest valence band shows a dispersion along the direction of layer stacking. However, MT shows almost no band dispersion along either direction because it has a significantly lower degree of polymerization than melon and low-molecularweight oligomers are loosely bonded to each other through hydrogen bonds to form the crystal structures. A slight overlap between the $\pi$-orbitals in neighboring oligomers is observed, which is needed for band dispersion. The energy gap based on the band calculation of MT is higher than that of melon by $\sim 0.1 \mathrm{eV}$, which is consistent with the energy gap evaluated from the shake-up satellites in the XPS data shown in Fig. 3.

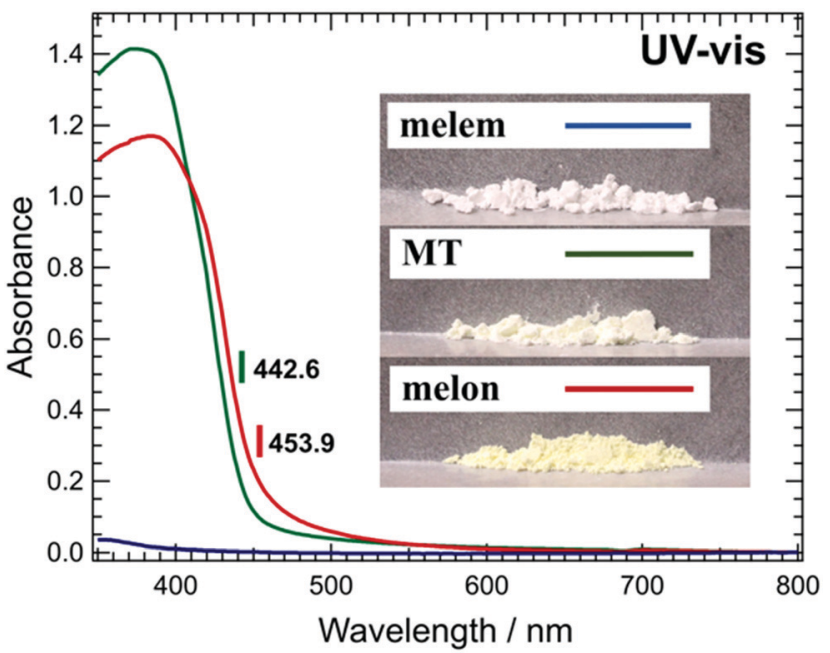

Fig. 5 UV-vis spectra of melem, MT, and melon prepared in $\mathrm{N}_{2}$ atmosphere. Photographs of each sample are displayed in the inset. Vertical bars indicate the wavelengths of the absorption edges, $\lambda_{\text {opt }}$.

Fig. 5 shows the UV-vis spectra of melon and MT synthesized in $\mathrm{N}_{2}$ atmosphere. The data for melem are also shown for reference. The spectrum of melem may not be accurately measured because of the PL of melem, but the absorption edge of its solution is at $\lambda_{\text {opt }}=339.1 \mathrm{~nm}$, as shown in Fig. S5 (ESI $\dagger$ ), which is in the shorter wavelength region than that shown in this figure. The absorption edges of melon and CN415 are located on the longer wavelength side in comparison to that of melem, and the absorption edge of MT $\left(\lambda_{\text {opt }}=442.6 \mathrm{~nm}\right)$ is located at a slightly shorter wavelength in comparison to that of melon $\left(\lambda_{\text {opt }}=453.9 \mathrm{~nm}\right)$, indicating that MT has a larger $E_{\mathrm{g}}$ than melon. This trend is in agreement with the energy gap variation caused by different polymerization temperatures, as previously reported in the literature. ${ }^{27,28}$ The colors of the samples observed in the photographs are consistent with the differences in the absorption edges observed in the UV-vis spectra. MT has a whitish color compared to that of melon. Furthermore, this result is consistent with the magnitude of $E_{\mathrm{g}}$ for melon and MT evaluated from the energies of the shake-up satellites in the XPS data shown in Fig. 3(a).

The PL spectra of the powder samples of melem, melon, and MT are shown in Fig. 6. Melem exhibits intense luminescence in the near-UV region. Both melon and melem tetramers show emission at longer wavelengths than melem due to a change in the electronic structure caused by the expansion of the $\pi$ conjugated system upon polymerization. A comparison of the spectra of melon and MT shows that the spectral shape at approximately $450 \mathrm{~nm}$ changes slightly. The wavelengths for maximum intensity of PL, $\lambda_{\mathrm{PL}}$, for melon and MT are $458.9 \mathrm{~nm}$ and $444.6 \mathrm{~nm}$, respectively. The intensity at approximately $500 \mathrm{~nm}$ is also different, and the peak width of MT is slightly narrower, as summarized in Table 1. The luminescent colors observed in the photographs for melon and MT are slightly different; the luminescence of MT is more bluish. In comparison to the emission wavelengths of oligomers such as dimers 


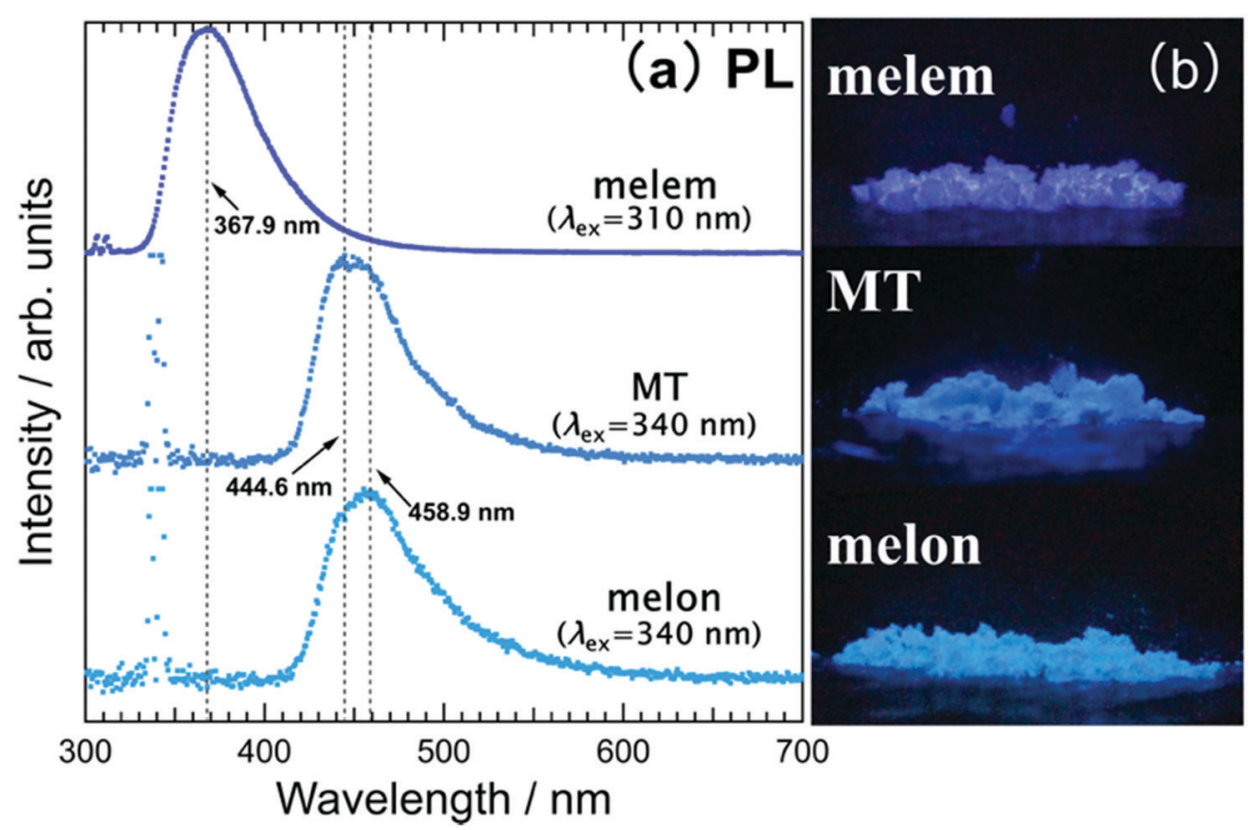

Fig. 6 (a) PL spectra of the powder samples of melem, MT, and melon measured at room temperature. (b) Photographs of the samples under ultraviolet (mercury lamp) irradiation. The excitation wavelength $\lambda_{\mathrm{ex}}$, which gives the highest quantum yield of each material, was used. The results of PL measurements using $\lambda_{\text {ex }}=310 \mathrm{~nm}$ and $340 \mathrm{~nm}$ for each material are shown in Fig. S6 (ESI $\dagger$ ).

Table 1 Optical properties of the powder samples of melem, melon, and MT. $\lambda_{\text {opt }}$ represents the wavelength of the absorption edge in the UV-vis absorption spectra. $\lambda_{\mathrm{PL}}, \eta$, and $\Delta \lambda_{\mathrm{PL}}$ are the wavelength at the maximum $\mathrm{PL}$ intensity, absolute quantum yield of $\mathrm{PL}$, and full width at half maximum (FWHM) of PL spectra, respectively

\begin{tabular}{lllrl}
\hline Sample & $\lambda_{\text {opt }} / \mathrm{nm}$ & $\lambda_{\text {PL }} / \mathrm{nm}$ & $\eta / \%$ & FWHM $/ \mathrm{nm}$ \\
\hline Melem & $(339.1)^{a}$ & 367.9 & 71.2 & 55.0 \\
MT (rinsed with DMSO) & 442.1 & 444.6 & 10.4 & 54.0 \\
Melon & 453.6 & 458.9 & 7.4 & 62.2
\end{tabular}

${ }^{a}$ As the absorption edge of melem is affected by the luminescence of melem in the powder form, the one dissolved in solvent was measured.

and trimers reported in previous studies, ${ }^{23} \mathrm{MT}$ emits at longer wavelengths, which further supports the existence of tetramers rather than small oligomers. Based on the quantum efficiency measurements, the absolute quantum yield, $\eta$, of MT is higher than that of melon, i.e., $\eta=7.4 \%$ for melon and $\eta=10.4 \%$ for MT. Considering the PL of MT, the color purity shows slight improvement, and the quantum efficiency is also enhanced with blue light emission. The high quantum efficiency of MT is probably related to the delayed fluorescence of melem. ${ }^{28,29}$ However, it is necessary to confirm that MT shows similar delayed fluorescence in the future studies. As MT can be isolated and is chemically stable under ambient conditions, it can be concluded that MT is a superior as a blue light-emitting material compared to melon, and can potentially be applied as a new luminescent material in optoelectronic devices.

The optical properties of the powder samples of melem, melon, and MT are summarized in Table 1. The absolute PL quantum yield of melem is very high, and the yield of MT, which has an electronic structure derived from that of an oligomer with four connected melem units, is also higher than that of melon.

Based on the above discussion, the thermal polymerization process of melamine as a precursor in $\mathrm{N}_{2}$ atmosphere is shown in Fig. 7. When melamine is heated at $415^{\circ} \mathrm{C}$ in $\mathrm{N}_{2}$ atmosphere, as-CN415 with XRD patterns different from those of melon and melem is obtained as a product. In addition, when as-CN415 is immersed in DMSO (solvent used for melem), a stable substance that remains insoluble is observed, which is identified as a melem tetramer. Melem tetramer is a new $\mathrm{CN}$ compound that has a unique crystal structure and chemical composition different from those of previously known CN-based compounds.

Melem and melon are converted into amorphous melon when heated at high temperatures. Amorphous melon is not completely amorphous. The XRD patterns of amorphous melon are similar to those of melon, but the intensities of the peaks are weaker, and the (002) diffraction peak in XRD data is broader than that of melon. Additionally, amorphous melon is thicker and yellow in color because of the defects generated by heating. ${ }^{30-33}$ The XRD data shown in Fig. S7 (ESI $\dagger$ ) indicate that as-CN415 and MT are also converted into amorphous melon by further heating at $415{ }^{\circ} \mathrm{C}$. It can be speculated that when reheated at $415{ }^{\circ} \mathrm{C}$, MT changes to melon, followed by a change to amorphous melon. It is expected that a polymerization reaction occurs in which the ends of the oligomer, which is a building unit of MT, are bonded.

\subsection{Characterization of $\mathrm{CN}$ compounds prepared in vacuum}

When thermal polymerization of melon was carried out under vacuum using as-CN415 as a precursor, melon and melem 


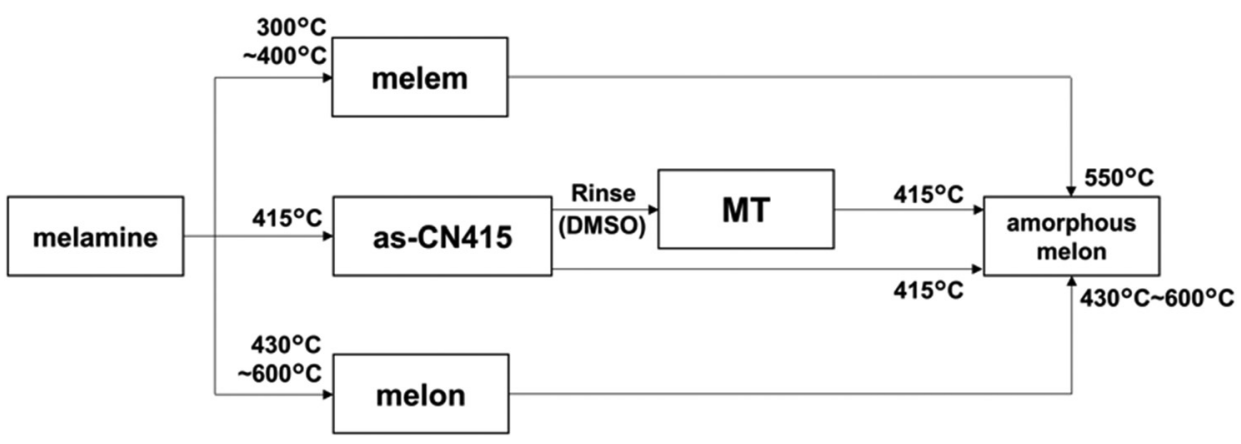

Fig. 7 Thermal polymerization of melamine as a precursor in $\mathrm{N}_{2}$ atmosphere.

tetramers were obtained; these were also obtained under atmospheric pressure of $\mathrm{N}_{2}$. However, the temperature dependence of the polymerization reactions was different from that under atmospheric pressure. Here, as-CN415 was obtained by heating melamine in $\mathrm{N}_{2}$ atmosphere (Fig. 7). The melem tetramer was obtained by reheating as-CN415 under vacuum because the unreacted melem remaining in the as-CN415 sublimates was treated by heating at $180{ }^{\circ} \mathrm{C}$ for four hours in vacuum, leaving only MT. MT could be removed from as-CN415 not only by rinsing with DMSO but also degassing under vacuum. A comparison of the XRD patterns of MT ("CN415" in Fig. 2) to that of Fig. 8 showed that the pattern of MT in Fig. 8 was identical to that in Fig. 2. In contrast, the intensity ratio of each peak was different even though the precursor (as-CN415) used was the same and preparation procedure was identical. This indicated that the crystallinity of the resulting MT was different when heated in $\mathrm{N}_{2}$ atmosphere and vacuum. Considering the XRD data shown in Fig. 2 and 8, the MT prepared under vacuum showed better crystallinity than that prepared in $\mathrm{N}_{2}$ atmosphere. The cause of this difference in the crystallinity remains

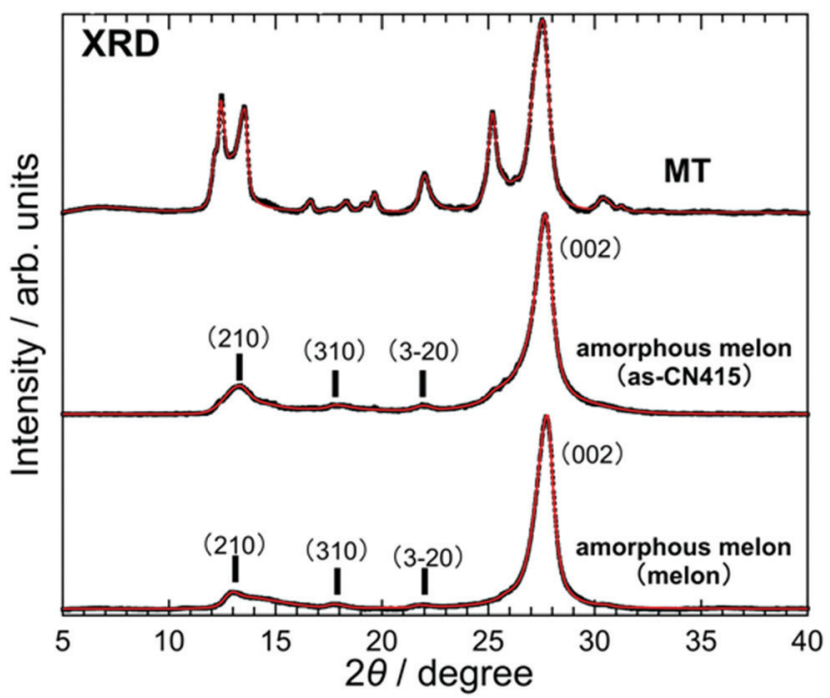

Fig. 8 (a) XRD profiles of the powder samples of MT prepared from asCN415 under vacuum and amorphous melon obtained by reheating asCN415 or melon in vacuum. unclear, but it is possible that DMSO molecules infiltrates MT when they are treated with DMSO, preventing effective restacking even after drying in $\mathrm{N}_{2}$ atmosphere.

Fig. 9 shows the UV-vis spectra of MT and amorphous melon. The samples of the latter were prepared via two different methods. The sample of amorphous melon corresponding to the middle spectrum was obtained by reheating as-CN415 at $230{ }^{\circ} \mathrm{C}$ for $5.3 \mathrm{~h}$ under vacuum, while the sample in the lower spectrum was obtained by reheating melon at $180{ }^{\circ} \mathrm{C}$ for $5.3 \mathrm{~h}$ under vacuum. In both cases, the absorption edge of amorphous melon is located at a longer wavelength than that of melon (Fig. 5) and MT. The thermal polymerization that occurs under vacuum should be identical as that under atmospheric $\mathrm{N}_{2}$ conditions. Amorphous melon has the same structure as that of melon. Thus, the results indicate that MT can be converted into melon by reheating under vacuum as well as atmospheric pressure in $\mathrm{N}_{2}$, but the temperature required for the former is significantly lower than that in the latter. Additionally, amorphous melon obtained by the reheating of melon

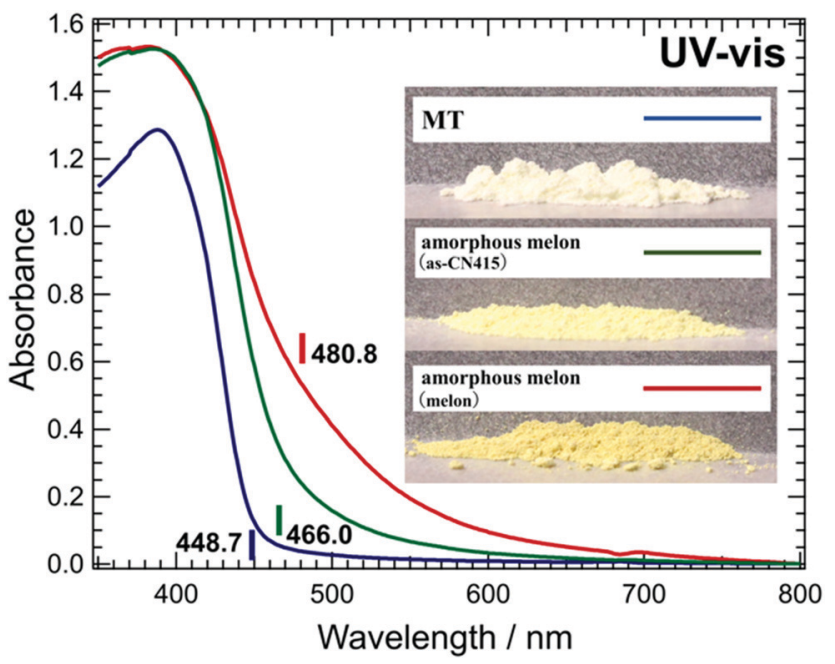

Fig. 9 UV-vis spectra of MT prepared from as-CN415 under vacuum and amorphous melon obtained by reheating as-CN415 or melon under vacuum. Calcination conditions to obtain amorphous melon from melon and as- $\mathrm{CN} 415$ are $230{ }^{\circ} \mathrm{C}$ for $5.3 \mathrm{~h}$ and $180{ }^{\circ} \mathrm{C}$ for $5.3 \mathrm{~h}$, respectively. Photographs of each sample are displayed in the inset. Vertical bars indicate the wavelengths of the absorption edges, $\lambda_{\text {opt }}$. 


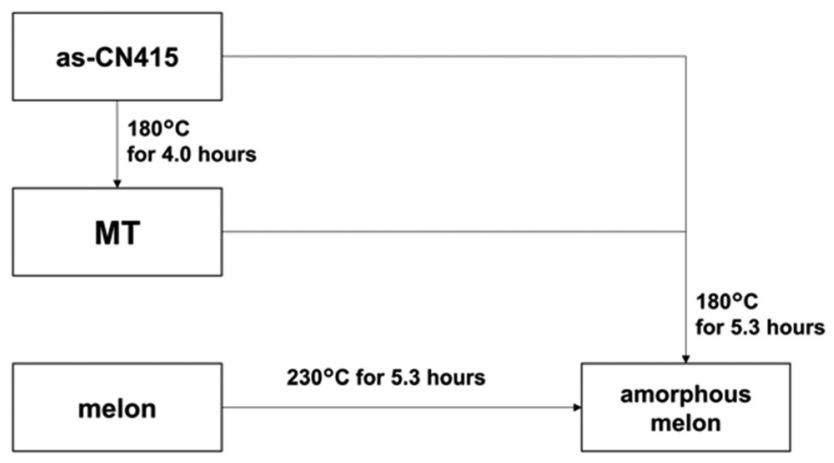

Fig. 10 Formation of amorphous melon under vacuum using as-CN415 as a starting material.

shows tailing toward the longer wavelength side up to $\lambda$ of $800 \mathrm{~nm}$. Such long tailing is not observed for amorphous melon obtained from as-CN415, which is also indicated by the difference in the colors of the samples in Fig. 8. A previous study reported that amorphous melon exhibited similar broad absorption over the entire visible spectrum. Kang et al. reported that the reheating of microcrystalline melon at $620{ }^{\circ} \mathrm{C}$ for two hours in Ar atmosphere afforded amorphous melon, which showed a wider light absorption range than melon and exhibited photocatalytic activity with excellent hydrogen generation. ${ }^{30-33}$

Based on the XRD and UV-vis spectroscopy data, the thermal polymerization process to generate amorphous melon from different compounds under vacuum is shown in Fig. 10. When as-CN415 is reheated at a low temperature of $180{ }^{\circ} \mathrm{C}$ for $4.0 \mathrm{~h}$ under vacuum, MT is obtained because of the sublimation of unreacted melem from as-CN415 under vacuum. Furthermore, when MT or as-CN415 is reheated at $180{ }^{\circ} \mathrm{C}$ for $5.3 \mathrm{~h}$ in vacuum, further polymerization occurs, and an identical crystal structure as that of amorphous melon that is formed when melon is reheated is obtained. These polymerization reactions are similar to those that occur at atmospheric pressure, but thermal polymerization occurs at a significantly lower temperature under vacuum than at atmospheric pressure. This can be explained as follows. As shown in the theoretical phase diagram, ${ }^{23}$ low ammonia partial pressure facilitates the desorption of ammonia during the process, and the reaction proceeds predominantly in the polymerization direction. Furthermore, low ammonia partial pressure is favorable for producing amorphous melon. Amorphous melon can be easily obtained at lower temperature under vacuum than that at atmospheric pressure because ammonia is easily desorbed during polymerization.

The PL spectra of the powder samples of MT and amorphous melon are shown in Fig. 11(a), while the photographs of the samples irradiated with ultraviolet light are exhibited in Fig. 11(b). Due to the polymerization of as-CN415 by heating under vacuum, the wavelength corresponding to the maximum luminescence intensity in the PL spectrum of amorphous melon is longer than that of MT by approximately $16 \mathrm{~nm}$, and the peak is broader. This corresponds to the color change in the PL image of the powder (Fig. 11(b)). As observed in the photographs shown in Fig. 11(b), the luminescence intensity decreases significantly as the polymerization progresses to afford amorphous melon, and the color purity degrades. While MT shows potential for application as a new material for blue light emission, amorphous melon can potentially show appropriate photocatalytic performance owing to its light absorption over a wide wavelength range in the visible region.

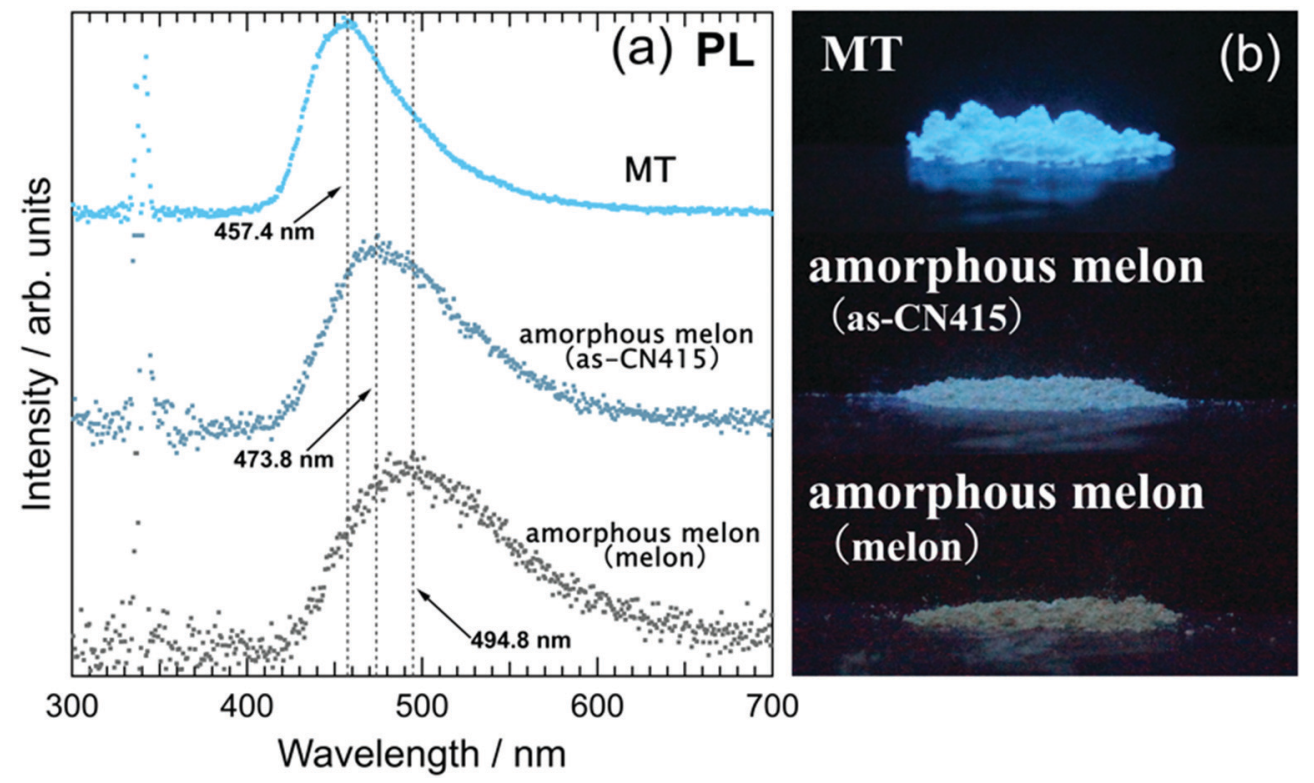

Fig. 11 (a) PL spectra of the powder samples of MT and amorphous melon at room temperature measured using excitation light of $\lambda_{\text {ex }}=340 \mathrm{~nm}$. Measurements for amorphous melon were performed on two types of samples prepared in the same manner as shown in Fig. 9. (b) Photographs of the samples under ultraviolet (mercury lamp) irradiation. 
Table 2 Optical properties of the powder samples of MT and amorphous melon. Amorphous melon samples were obtained by reheating as-CN415 at $180{ }^{\circ} \mathrm{C}$ for $5.3 \mathrm{~h}$ under vacuum and melon at $230{ }^{\circ} \mathrm{C}$ for $5.3 \mathrm{~h}$. $\lambda_{\text {opt }}$ represents the wavelength of the absorption edge in the UV-vis absorption spectra. $\lambda_{\mathrm{PL}} \eta$, and $\Delta \lambda_{\mathrm{PL}}$ are the wavelength at the maximum PL intensity, absolute quantum yield of $\mathrm{PL}$, and FWHM of PL spectra, respectively

\begin{tabular}{lllrc}
\hline Sample & $\begin{array}{l}\lambda_{\text {opt }} / \\
\mathrm{nm}\end{array}$ & $\begin{array}{l}\lambda_{\mathrm{PL}} / \\
\mathrm{nm}\end{array}$ & \multicolumn{1}{c}{$\eta / \%$} & \multicolumn{1}{c}{$\begin{array}{l}\text { FWHM/ } \\
\mathrm{nm}\end{array}$} \\
\hline MT (as-CN415 heated 4.0 h) & 448.7 & 457.4 & 13.6 & 64.0 \\
MT (as-CN415 heated 5.3 h) & 466.0 & 473.8 & 4.3 & 86.0 \\
Amorphous melon (melon & 480.8 & 494.8 & 1.9 & 109.8 \\
heated 5.3 h) & & & &
\end{tabular}

The parameters that describe the optical properties of MT and amorphous melon are listed in Table 2. Compared to the properties of MT included in Table 1, the PL wavelength of MT prepared under vacuum is slightly red-shifted, and the width of the PL peak is increased because heating under vacuum causes the terminal amino group to desorb from MT, introducing a small amount of defects. However, the quantum yield of MT prepared under vacuum is higher. Therefore, a comparison of the photograph of MT in Fig. 11(b) to the photograph of MT in Fig. 6(b) shows that the former is brighter and glows with a lighter blue color. In contrast, significantly diminished PL is observed for amorphous melon obtained from as-CN415 or melon. This remarkable change is speculated to be due to a large number of carrier traps created in amorphous melon, which facilitate the dissociation of excitons generated by light absorption and promote charge separation. This interpretation is consistent with the superior photocatalytic activity of amorphous melon compared to that of melon, as reported in previous studies. ${ }^{30-33}$

\section{Conclusion}

After careful reexamination of the known calcination conditions of melon, a new CN compound, MT, was identified in this study. MT thermally polymerizes at a temperature slightly lower than that used for the thermal polymerization of melon. The structure and physical properties of MT are determined using XRD, FT-IR, XPS, UV-vis, and PL analyses.

MT is formed during the polymerization of melamine to melon and has a lower degree of polymerization than melon. Analysis of the N 1s core level XPS data of MT suggests that it has a crystal structure in which an oligomer consisting of four melem units forms a building unit. The proposed crystal structure of MT is consistent with the XRD, UV-vis, and PL analyses. Notable characteristics of MT include a higher absolute PL quantum yield than melon and blue light emission with improved color purity. MT prepared by reheating as-CN415 under vacuum affords higher crystallinity and fluorescence quantum yield than MT prepared under $\mathrm{N}_{2}$ atmosphere.

This study shows that during the thermal polymerization of CN compounds, valuable new CN compounds can possibly be obtained by careful examination of the calcination conditions. $\mathrm{CN}$ compounds with various structures can be formed because of the coexistence of strong $\mathrm{CN}$ bonds and weak intermolecular forces such as hydrogen bonds and dispersion forces as cohesive forces.

\section{Conflicts of interest}

There are no conflicts to declare.

\section{Acknowledgements}

The authors are grateful to Prof. M. Tamura of the Tokyo University of Science for his assistance with FT-IR measurements.

\section{References}

1 A. Fujishima and K. Honda, Electrochemical photolysis of water at a semiconductor electrode, Nature, 1972, 238(5358), 37-38, DOI: $10.1038 / 238037 \mathrm{a} 0$.

2 A. K. P. Mann, E. M. P. Steinmiller and S. E. Skrabalak, Elucidating the structure-dependent photocatalytic properties of $\mathrm{Bi}_{2} \mathrm{WO}_{6}$ : A synthesis guided investigation, Dalton Trans., 2012, 41(26), 7939, DOI: 10.1039/c2dt30097d.

3 N. N. Vu, S. Kaliaguine and T. O. Do, Critical aspects and recent advances in structural engineering of photocatalysts for sunlight-driven photocatalytic reduction of $\mathrm{CO}_{2}$ into fuels, Adv. Funct. Mater., 2019, 29(31), 1-44, DOI: 10.1002/ adfm.201901825.

4 Q. Liang, Y. Shi, W. Ma, Z. Li and X. Yang, Enhanced photocatalytic activity and structural stability by hybridizing $\mathrm{Ag}_{3} \mathrm{PO}_{4}$ nanospheres with graphene oxide sheets, Phys. Chem. Chem. Phys., 2012, 14(45), 15657-15665, DOI: 10.1039/c2cp42465g.

5 W. Zhang, Y. Wang, Z. Wang, Z. Zhong and R. Xu, Highly efficient and noble metal-free NiS/CdS photocatalysts for $\mathrm{H}_{2}$ evolution from lactic acid sacrificial solution under visible light, Chem. Commun., 2010, 46(40), 7631-7633, DOI: 10.1039/c0cc01562h.

6 X. Wang, K. Maeda, A. Thomas, K. Takanabe, G. Xin, J. M. Carlsson, K. Domen and M. Antonietti, A metal-free polymeric photocatalyst for hydrogen production from water under visible light, Nat. Mater., 2009, 8(1), 76-80, DOI: $10.1038 /$ nmat2317.

7 Y. Wang, X. Wang and M. Antonietti, Polymeric graphitic carbon nitride as a heterogeneous organocatalyst: From photochemistry to multipurpose catalysis to sustainable chemistry, Angew. Chem., Int. Ed., 2012, 51(1), 68-89, DOI: 10.1002/anie.201101182.

$8 \mathrm{~J}$. Wang, C. Liu, S. Yang, X. Lin and W. Shi, Fabrication of a ternary heterostructure $\mathrm{BiVO}_{4}$ quantum dots $/ \mathrm{C}_{60} / \mathrm{g}-\mathrm{C}_{3} \mathrm{~N}_{4}$ photocatalyst with enhanced photocatalytic activity, J. Phys. Chem. Solids, 2020, 136(June 2019), 109164, DOI: 10.1016/j.jpcs.2019.109164.

9 S. Yang, C. Liu, J. Wang, X. Lin, Y. Hong, F. Guo and J. Shi, Enhanced photocatalytic activity of G- $\mathrm{C}_{3} \mathrm{~N}_{4}$ quantum dots/ 
$\mathrm{Bi}_{3.64} \mathrm{Mo}_{0.36} \mathrm{O}_{6.55}$ nanospheres composites, J. Solid State Chem., 2020, 287, 121347, DOI: 10.1016/j.jssc.2020.121347.

10 Y. Xu, Q. Guo, L. Huang, H. Feng, C. Zhang, H. Xu and M. Wang, Toward efficient preconcentrating photocatalysis: $3 \mathrm{D}$ g- $\mathrm{C}_{3} \mathrm{~N}_{4}$ monolith with isotype heterojunctions assembled from hybrid 1D and 2D nanoblocks, ACS Appl. Mater. Interfaces, 2019, 11(35), 31934-31942, DOI: 10.1021/acsami.9b09290.

11 L. Wang, Y. Hong, E. Liu, Z. Wang, J. Chen, S. Yang, J. Wang, $\mathrm{X}$. Lin and J. Shi, Rapid polymerization synthesizing highcrystalline g- $\mathrm{C}_{3} \mathrm{~N}_{4}$ towards boosting solar photocatalytic $\mathrm{H}_{2}$ generation, Int. J. Hydrogen Energy, 2020, 45(11), 6425-6436, DOI: 10.1016/j.ijhydene.2019.12.168.

12 W. J. Ong, L. K. Putri and A. R. Mohamed, Rational design of carbon-based 2D nanostructures for enhanced photocatalytic $\mathrm{CO}_{2}$ reduction: A dimensionality perspective, Chem. - Eur. J., 2020, 26(44), 9710-9748, DOI: 10.1002/chem.202000708.

13 K. Akaike, K. Aoyama, S. Dekubo, A. Onishi and K. Kanai, Characterizing electronic structure near the energy gap of graphitic carbon nitride based on rational interpretation of chemical analysis, Chem. Mater., 2018, 30(7), 2341-2352, DOI: 10.1021/acs.chemmater.7b05316.

14 J. Liebig, Uber einige stickstoff - verbindungen, Ann. Pharm., 1834, 10(1), 1-47, DOI: 10.1002/jlac.18340100102.

15 H. Inoki, G. Seo and K. Kanai, Synthesis of graphitic carbon nitride under low ammonia partial pressure, Appl. Surf. Sci., 2020, 534, 147569, DOI: 10.1016/j.apsusc.2020.147569.

16 K. Aoyama, K. Akaike and K. Kanai, Electroluminescence from sodium-doped polymeric carbon nitride film, Chem. Phys. Lett., 2020, 749, 137475, DOI: 10.1016/j.cplett.2020.137475.

17 H. Arazoe, D. Miyajima, K. Akaike, F. Araoka, E. Sato, T. Hikima, M. Kawamoto and T. Aida, An autonomous actuator driven by fluctuations in ambient humidity, Nat. Mater., 2016, 15(10), 1084-1089, DOI: 10.1038/nmat4693.

18 B. Jürgens, E. Irran, J. Senker, P. Kroll, H. Müller and W. Schnick, Melem (2,5,8-triamino-tri-s-triazine), an important intermediate during condensation of melamine rings to graphitic carbon nitride: Synthesis, structure determination by X-ray powder diffractometry, solid-state NMR, and theoretical studies, J. Am. Chem. Soc., 2003, 125(34), 10288-10300, DOI: 10.1021/ja0357689.

19 F. Fina, S. K. Callear, G. M. Carins and J. T. S. Irvine, Structural Investigation of Graphitic Carbon Nitride via XRD and Neutron Diffraction, Chem. Mater., 2015, 27(7), 2612-2618, DOI: 10.1021/acs.chemmater.5b00411.

20 X. Yuan, K. Luo, N. Liu, X. Ji, C. Liu, J. He, G. Tian, Y. Zhao and D. Yu, Cluster-model DFT simulations of the infrared spectra of triazine-based molecular crystals, Phys. Chem. Chem. Phys., 2018, 20(32), 20779-20784, DOI: 10.1039/c8cp01550c.

21 T. Komatsu, Attempted chemical synthesis of graphite-like carbon nitride, J. Mater. Chem., 2001, 11(3), 799-801, DOI: 10.1039/b007673m.

22 T. Tyborski, C. Merschjann, S. Orthmann, F. Yang, M. C. Lux-Steiner and T. Schedel-Niedrig, Crystal structure of polymeric carbon nitride and the determination of its process-temperature-induced modifications, J. Phys. Condens. Matter, 2013, 25(39), 395402, DOI: 10.1088/09538984/25/39/395402.

23 T. Botari, W. P. Huhn, V. W. H. Lau, B. v. Lotsch and V. Blum, Thermodynamic equilibria in carbon nitride photocatalyst materials and conditions for the existence of graphitic carbon nitride $\mathrm{G}^{-} \mathrm{C}_{3} \mathrm{~N}_{4}$, Chem. Mater., 2017, 29(10), 4445-4453, DOI: 10.1021/acs.chemmater.7b00965.

24 V. W. H. Lau, M. B. Mesch, V. Duppel, V. Blum, J. Senker and B. V. Lotsch, Low-molecular-weight carbon nitrides for solar hydrogen evolution, J. Am. Chem. Soc., 2015, 137(3), 1064-1072, DOI: 10.1021/ja511802c.

25 J. Wen, R. Li, R. Lu and A. Yu, Photophysics and photocatalysis of melem: A spectroscopic reinvestigation, Chem. Asian J., 2018, 13(8), 1060-1066, DOI: 10.1002/asia. 201800186.

26 H. Zheng, Z. Zhao, J. B. Phan, H. Ning, Q. Huang, R. Wang, J. Zhang and W. Chen, Highly efficient metal-free twodimensional luminescent melem nanosheets for bioimaging, ACS Appl. Mater. Interfaces, 2020, 12(2), 2145-2151, DOI: 10.1021/acsami.9b19915.

27 P. J. Larkin, M. P. Makowski and N. B. Colthup, The form of the normal modes of $s$-triazine: Infrared and Raman spectral analysis and $a b$ initio force field calculations, Spectrochim. Acta, Part A, 1999, 55(5), 1011-1020, DOI: 10.1016/ S1386-1425(98)00244-3.

28 J. Wen, R. Li, R. Lu and A. Yu, Photophysics and photocatalysis of melem: A spectroscopic reinvestigation, Chem. Asian J., 2018, 13(8), 1060-1066, DOI: 10.1002/asia. 201800186.

29 H. B. Zheng, W. Chen, H. Gao, Y. Y. Wang, H. Y. Guo, S. Q. Guo, Z. L. Tang and J. Y. Zhang, Melem: An efficient metal-free luminescent material, J. Mater. Chem. C, 2017, 5(41), 10746-10753, DOI: 10.1039/c7tc02966g.

30 V. N. Khabashesku, J. L. Zimmerman and J. L. Margrave, Powder synthesis and characterization of amorphous carbon nitride, Chem. Mater., 2000, 12(11), 3264-3270, DOI: 10.1021/cm000328r.

31 Z. Liu, G. Wang, H. S. Chen and P. Yang, An amorphous/ crystalline $\mathrm{g}-\mathrm{C}_{3} \mathrm{~N}_{4}$ homojunction for visible light photocatalysis reactions with superior activity, Chem. Commun., 2018, 54(37), 4720-4723, DOI: 10.1039/c8cc01824c.

32 Y. Kang, Y. Yang, L. C. Yin, X. Kang, G. Liu and H. M. Cheng, An amorphous carbon nitride photocatalyst with greatly extended visible-light-responsive range for photocatalytic hydrogen generation, Adv. Mater., 2015, 27(31), 4572-4577, DOI: $10.1002 /$ adma.201501939.

33 Q. Tay, P. Kanhere, C. F. Ng, S. Chen, S. Chakraborty, A. C. H. Huan, T. C. Sum, R. Ahuja and Z. Chen, Defect engineered $\mathrm{G}-\mathrm{C}_{3} \mathrm{~N}_{4}$ for efficient visible light photocatalytic hydrogen production, Chem. Mater., 2015, 27(14), 4930-4933, DOI: 10.1021/acs.chemmater.5b02344. 